\title{
PAPER \\ Performance Analysis of a Cognitive Radio Network with Imperfect Spectrum Sensing
}

\author{
Osama SALAMEH $^{\dagger, \dagger \dagger \mathrm{a})}$, Koen DE TURCK ${ }^{\dagger \dagger \dagger \mathrm{b})}$, Dieter FIEMS ${ }^{\dagger \mathrm{c})}$, Herwig BRUNEEL ${ }^{\dagger \mathrm{d})}$, Nonmembers, , \\ and Sabine WITTEVRONGEL ${ }^{\dagger \mathrm{e})}$, Member
}

\begin{abstract}
SUMMARY In Cognitive Radio Networks (CRNs), spectrum sensing is performed by secondary (unlicensed) users to utilize transmission opportunities, so-called white spaces or spectrum holes, in the primary (licensed) frequency bands. Secondary users (SUs) perform sensing upon arrival to find an idle channel for transmission as well as during transmission to avoid interfering with primary users (PUs). In practice, spectrum sensing is not perfect and sensing errors including false alarms and misdetections are inevitable. In this paper, we develop a continuous-time Markov chain model to study the effect of false alarms and misdetections of SUs on several performance measures including the collision rate between PUs and SUs, the throughput of SUs and the SU delay in a CRN. Numerical results indicate that sensing errors can have a high impact on the performance measures. key words: cognitive radio, opportunistic scheduling, Markov chain, performance analysis
\end{abstract}

\section{Introduction}

The introduction of Cognitive Radio Networks (CRNs) aims at providing a solution to the problem of wireless spectrum scarcity [1]-[3]. Under the CRN paradigm there is a secondary network that operates parallel to a primary network, where the secondary users (SUs) make use of the same channels as the primary users (PUs). The PUs are licensed users and can occupy any channel not used by another PU. The main idea of CRNs is to allow SUs to opportunistically use the channels not currently occupied by the PUs, thereby increasing the overall utilization of the channels. We assume that the primary and secondary networks are completely independent of each other. There is no coordination between them. This makes it necessary for a SU to perform channel sensing before transmission in order not to interfere with a possible PU occupying the channel. For an overview of sensing techniques, we refer to the papers [4]-[7] and the

Manuscript received January 26, 2017.

Manuscript revised May 24, 2017.

Manuscript publicized June 22, 2017.

$\dagger$ The authors are with the Department of Telecommunications and Information Processing (TELIN), Ghent University, Ghent, Belgium.

${ }^{\dagger}$ The author is with the Faculty of Engineering and Information Technology, Arab American University, Jenin, Palestine.

${ }^{\dagger \dagger}$ The author is with the Laboratoire des Signaux et Systèmes, CentraleSupélec, Gif-sur-Yvette, France.

a)E-mail: osama.salameh@aauj.edu

b) E-mail: koen.deturck@ centralesupelec.fr

c) E-mail: Dieter.Fiems@UGent.be

d)E-mail: Herwig.Bruneel@UGent.be

e) E-mail: Sabine.Wittevrongel@UGent.be DOI: 10.1587/transcom.2017EBP3037 references therein. In addition, a SU needs to sense an acquired channel during transmission in order to evacuate the channel upon the arrival of a PU on the channel.

In the ideal scenario, the PUs are not affected by the presence of SUs. In order to ensure this, the SUs should have perfect sensing abilities. In practice, sensing errors are inevitable including SU false alarms (where a SU wrongly thinks there is a PU on a channel) and SU misdetections of PUs (where a SU fails to detect the actual presence of a $\mathrm{PU}$ on a channel). Both types of sensing errors are possible while searching for an idle channel upon arrival and during transmission. Based on a false alarm, a SU fails to make use of a transmission opportunity. A SU misdetection of a PU occupying a channel is a more serious error as it results in a collision between a SU and a PU, thus degrading the performance of both primary and secondary networks.

In this paper, we present a generic study of the effect of sensing errors on the performance of the primary and secondary networks. We consider two classes of misdetection and two types of false alarm. The first class of misdetection occurs when a sensing SU incorrectly considers a channel that is occupied by a PU as an idle channel and starts to transmit. The second class of misdetection occurs when a transmitting SU fails to detect the arrival of a PU and does not evacuate the transmission channel. These classes are denoted as class-A and class-B misdetection respectively. They both result in a collision between a SU and a PU and we assume that the collided SU and PU are dropped from the system. Type-I false alarm occurs when a sensing SU fails to detect an idle channel and type-II false alarm occurs when a transmitting SU evacuates a channel without a PU arrival. They both result in a lost transmission opportunity. Our aim is to gain insight in the CRN performance for a wide range of values of the false alarm and misdetection parameters.

The CRN performance analysis in this paper makes use of the tool of continuous-time Markov chain (CTMC) modeling. CTMCs are widely used for modeling systems involving stochastic phenomena in various application domains. In particular, CTMCs play an important role in the design and performance evaluation of telecommunication systems and networks ever since the early work of A.K. Erlang in the field of telephone networks analysis [8]. The attractiveness of CTMCs lies in their defining feature that given the present system state, the future is independent of the past, better known as the Markov property. For further background on the theory of CTMCs and their applica- 
tions we refer the reader to the books [9]-[12]. The specific CTMC model established in the present paper keeps track of a three-dimensional system state consisting of the numbers of transmitting PUs, transmitting SUs en sensing SUs. Based on this CTMC model we derive various performance measures of the system, such as the throughputs of PUs and SUs and the collision rate between PUs and SUs.

The remainder of the paper is organized as follows. In Sect. 2, we discuss related literature on mathematical models for CRNs that account for sensing. The system under study is described in detail in Sect. 3 and the corresponding CTMC model is presented in Sect. 4. Several performance measures are derived in Sect. 5. Numerical results are presented and discussed in Sect. 6 and the paper is concluded in Sect. 7.

\section{Related Work}

CRNs with sensing errors have been studied extensively using different mathematical tools. In [13], load balancing for SUs on the different CRN channels is studied using a preemptive M/G/1 queueing model. An optimal number of channels for sensing by a SU is obtained (in view of the balance between the difficulty for a SU to find an idle channel and the amount of time wasted on sensing), where false alarms and misdetections are considered. In [14], the sensing-throughput tradeoff for CRNs is studied and the optimal sensing time that results in the highest throughput for SUs is obtained using Monte Carlo simulation. Both perfect and imperfect sensing are considered in [15], where an optimal sensing-transmission structure is presented to maximize the SU spectrum usage while providing a satisfactory level of protection for PUs. However, the analysis in [15] is limited to one PU channel only. In [16], stochastic network calculus is used to analyze a CRN with imperfect sensing. Therein, the backlog and delay bounds are obtained. However, false alarms and misdetection of a PU by a SU are only considered for arriving SUs. The sensing time is considered negligible, while in practice it is not when compared to the transmission time [15].

The sensing time is taken into account in [17]. The utilization of PUs and SUs and the probability of collision between a PU and a SU are derived. The PU traffic is modeled by an on-off CTMC and the SU network is time-slotted. However, the analysis is limited to one channel only and sensing errors during a SU transmission are not considered. The cross-layer modeling of a multichannel CRN with imperfect sensing is presented in [18]. The throughput of SUs and the delay of PUs are analyzed. The primary and secondary networks are time-slotted and synchronized, which might be expensive to implement when the PU network is already operational [19]. In this case, SUs sense at the beginning of a time slot and sensing errors during SU transmission are not applicable.

Discrete-time Markov chains (DTMCs) are used to analyze the performance of CRNs in [20], [21]. In [20], the effect of false alarms and misdetections on the performance of a cooperative CRN is investigated. The throughput of
SUs is derived when the channels are assumed to experience slow Rayleigh fading. However, the sensing time is not taken into account and sensing errors during a SU transmission are not considered in [20]. CRNs with sensing errors are also studied in [21]. Therein, the focus is on the tradeoff between false alarms and misdetections and an adequate operating point of the sensing mechanism is chosen.

The analysis of CRNs with sensing errors using continuous-time Markov chains is considered in [22]-[27]. In [22], the performance measures include the PU termination probability, i.e., the probability that a $\mathrm{PU}$ is terminated due to a collision with a SU, and the SU success probability. One limitation of this study is the assumption that only an arriving SU can experience a collision with a PU and a transmitting SU will always correctly detect PU arrivals and start to search for a new idle channel. Other limitations are that the SU sensing time and false alarms for transmitting SUs are considered negligible and the number of channels is at most 3. In [23], the authors consider an extension where an arbitrary number of channels is possible but all the other limitations of [22] still hold. The analysis of a multichannel $\mathrm{CRN}$ is presented in [24], where the probability mass functions of the SU queue length and the SU queueing delay are obtained. Sensing errors are considered during the sensing interval, but the interaction between SUs and PUs in the CRN is assumed to be collision-free. The studies [22]-[24] all have in common that they do not take sensing errors of transmitting SUs into account.

Sensing errors of both sensing and transmitting SUs are considered in [25]-[27]. However, these studies have several other limitations including the absence of sensing time and the assumption that a transmitting SU can instantly switch to another channel if one is available upon a PU arrival. The effect of SU interruptions is only considered when no idle channels are available in the system and in this case an interrupted SU is placed in a virtual queue. It is assumed that SUs in the virtual queue cannot experience type-I false alarms or class-A misdetections. Moreover, it is assumed that collisions are not affected by the number of transmitting SUs in the system. Apart from these existing studies, the CTMC model considered in this paper takes the sensing time and the number of transmitting SUs into account. Also, our model fully captures the effect of interruptions, where every interrupted SU starts to sense the channels to find a new transmission opportunity and consequently we consider the possibility of false alarms and misdetections at every stage of a SU's existence in the system. The present paper is an extension of our previous work [28], where perfect spectrum sensing was assumed.

\section{System under Study}

As illustrated in Fig. 1, we consider a CRN where a secondary user network operates parallel to a primary user network. The wireless spectrum is divided into $N$ error-free frequency bands (channels) with the same bandwidth and the channels are not time-slotted. The primary user network 


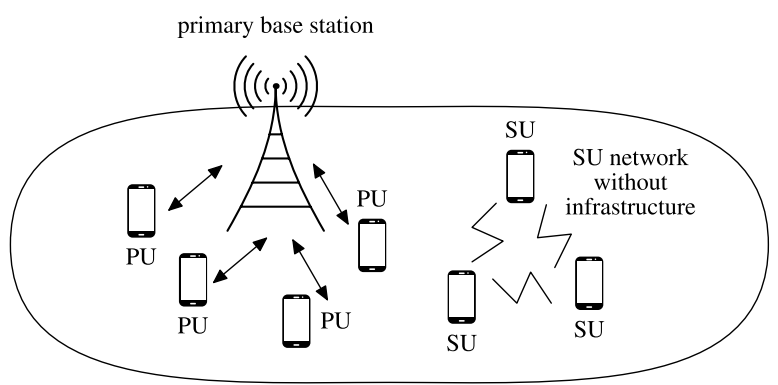

Fig. 1 CRN scenario with primary and secondary networks.
Table 1 System parameters.

\begin{tabular}{ll}
\hline$N$ & number of channels \\
$\lambda_{1}$ & PU arrival rate \\
$\lambda_{2}$ & SU arrival rate \\
$\mu_{1}$ & PU channel holding rate \\
$\mu_{2}$ & SU channel holding rate \\
$K$ & maximum number of sensing SUs \\
$\sigma$ & channel sensing rate \\
$p_{m 1}$ & class-A misdetection probability \\
$p_{m 2}$ & class-B misdetection probability \\
$p_{f 1}$ & type-I false alarm probability \\
$\delta_{f 2}$ & type-II false alarm rate \\
\hline
\end{tabular}

is an infrastructure-based network where a primary base station coordinates the access of PUs to the available frequency channels, so no collisions between PUs can occur. Specifically, to each arriving PU wanting to transmit data a channel for transmission is assigned randomly, provided there is at least one channel available (i.e., not already occupied by another PU); otherwise, if all channels are already in use by other PUs, the arriving PU is blocked. It is important to note that as soon as a channel is assigned to a PU, the PU starts to transmit immediately, i.e., PUs do not perform spectrum sensing. Also, the primary base station views all channels occupied by SUs as available channels and can assign PUs to them.

SUs are allowed to opportunistically use licensed frequency channels that are temporarily not occupied by PUs. The secondary user network is a network without infrastructure composed of a set of SUs equipped with cognitive capabilities, where each SU determines whether a channel is occupied or idle based on its own measurements. Furthermore, SUs can coordinate the access to the idle channels with other SUs by exchanging messages on a common control channel [25]; a SU is thus aware of the channels occupied by other SUs and there are no collisions between SUs, as in [26], [27]. Similarly to the primary network, the secondary network is not time-slotted. SUs perform spectrum sensing upon arrival to find an idle channel for transmission as well as during transmission to avoid the interference with PUs. As in [30]-[32], we consider the case where each SU operates in full duplex mode, i.e., a SU can sense a channel continuously while transmitting; for an implementation of a SU operating in full duplex mode we refer to [25]. Note that channel sensing of SUs during transmission has a positive impact on the primary network as it decreases the collision probability between a transmitting SU and an arriving PU; it moreover has a positive impact on the secondary network since no white space part is wasted on sensing. This is in contrast with the case where SUs do not operate in full duplex mode and a SU has to transmit and sense in cycles until the SU transmission is complete.

The system parameters are summarized in Table 1. In our analysis, we assume that PUs and SUs having data to transmit arrive according to independent Poisson processes with arrival rates $\lambda_{1}$ and $\lambda_{2}$ respectively. The channel holding times of PUs and SUs are modeled as exponentially distributed random variables with mean values $1 / \mu_{1}$ and $1 / \mu_{2}$, i.e., with channel holding rates $\mu_{1}$ and $\mu_{2}$, respectively.

An arriving SU starts sensing to determine an idle channel for transmission if there are less than a given maximum number $K$ of sensing SUs in the system; otherwise, the arriving SU is blocked. The maximum number of sensing SUs is referred to as the "sensing room size" in the sequel. As in [18], the sensing time is defined as the time to sense one channel that is selected randomly and we assume the sensing time is exponentially distributed with rate $\sigma$. The assumption that the sensing time is not fixed has also been used in e.g. [29].

When searching for an idle channel, a SU senses all channels not occupied by other SUs, i.e., both idle channels and those occupied by PUs. The outcome of the sensing of a channel by a SU is then one of the following results:

- the channel is found occupied by a PU correctly,

- the channel is found occupied by a PU while it is idle,

- the channel is found idle while it is occupied by a PU,

- the channel is found idle correctly.

In the first two cases, the SU randomly picks a channel and starts to sense again, where in case 2 a type-I false alarm is fired because of a lost transmission opportunity. Case 3 corresponds to a class-A misdetection of a PU and both users are dropped from the system due to collision. In case 4 the SU starts transmission.

Each SU transmits on one channel only and, as mentioned above, performs continuous sensing during transmission to detect a possible PU arrival on the channel. During this sensing one of the following (instantaneous) events can occur, causing a change in the system:

- a PU arrives on the channel and it is correctly detected,

- the SU wrongly thinks there is a PU arrival on the channel,

- a PU arrives on the channel and it is not detected.

In the first two cases, the SU goes back to sensing in order to search for an idle channel if the sensing room is not full and the SU is lost otherwise, where in case 2 a type-II false alarm is fired. Case 3 corresponds to a class-B misdetection of a PU and the collided PU and SU are dropped from the system. Note that as long as during the sensing none of the above 3 events occurs, the SU is still correctly detecting there is no PU arrival on the channel and simply continues its transmission without any change in the system. 
The probabilities of class-A and class-B misdetection of a PU by a SU are denoted by $p_{m 1}$ and $p_{m 2}$ respectively. Here, $p_{m 1}$ is the probability that a $\mathrm{SU}$ who is searching for an idle channel and has finished sensing a busy channel, has failed to detect the presence of a PU on that channel, while $p_{m 2}$ is the probability that the arrival of a PU to a channel occupied by a transmitting SU is not detected by that SU. We assume that a type-I false alarm occurs with probability $p_{f 1}$, i.e., $p_{f 1}$ is the probability that a $\mathrm{SU}$ who is searching for an idle channel and has finished sensing an idle channel, wrongly thinks the channel is occupied by a PU and thus fires a type-I false alarm. Like in [25], a transmitting SU wrongly thinks there is a PU arrival and thus fires a type-II false alarm according to a Poisson process with rate $\delta_{f 2}$. Note that the occurrence of type-II false alarms is not modeled by a probability, but by the rate $\delta_{f 2}$. This is because we assume that a transmitting SU performs continuous sensing and consequently can fire a type-II false alarm at any moment based on channel measurement results, where during an infinitesimal time interval of length $\mathrm{d} t$ a type-II false alarm occurs with probability $\delta_{f 2} \mathrm{~d} t$.

\section{CTMC Model}

In view of the non-time-slotted nature of both the primary and the secondary network, continuous-time Markov chain modeling is the natural modeling framework to investigate the performance of the considered cognitive radio system. In particular, in this section, we create a three-dimensional CTMC model of the system under study. To this end, we let system state $x$ correspond to the triplet $x=\left(x_{1}, x_{2}, x_{3}\right)$, where $x_{1}$ is the number of transmitting PUs, $x_{2}$ is the number of transmitting SUs and $x_{3}$ is the number of sensing SUs searching for an idle channel. The state space $S$ of the CTMC contains all possible system states. Clearly, $S$ is finite and contains all states $\left(x_{1}, x_{2}, x_{3}\right)$ such that

$$
0 \leq x_{1} \leq N, 0 \leq x_{2} \leq N-x_{1} \text { and } 0 \leq x_{3} \leq K .
$$

The next step is now to determine the infinitesimal generator or transition rate matrix $Q$ of the CTMC. A basic property of this (square) matrix is that its diagonal elements $q_{x, x}(x \in S)$ are such that the row sums of $Q$ are equal to zero. Another property is that the non-diagonal element $q_{x, y}$ $(x \in S, y \in S, x \neq y)$ of $Q$ is the transition rate from state $x$ to another state $y$. More explicitly, this means that given the CTMC is in system state $x$, a transition from state $x$ to another state $y(x \neq y)$ will occur in an infinitesimal time interval of length $\mathrm{d} t$ with probability $q_{x, y} \mathrm{~d} t$. In what follows, we establish expressions for these transition rates $q_{x, y}$ $(x \neq y)$. To do so, for each state $x=\left(x_{1}, x_{2}, x_{3}\right) \in S$ we need to consider all possible events that cause a transition out of state $x$ to another state $y(x \neq y)$ and to determine the corresponding rates. As an illustration, for a system state $x=\left(x_{1}, x_{2}, x_{3}\right)$ with $0<x_{1}<N, 0<x_{2}<N$ and $0<x_{3}<K$, the state transitions out of this state are depicted in Fig. 2.

To list the different events that cause a change of the

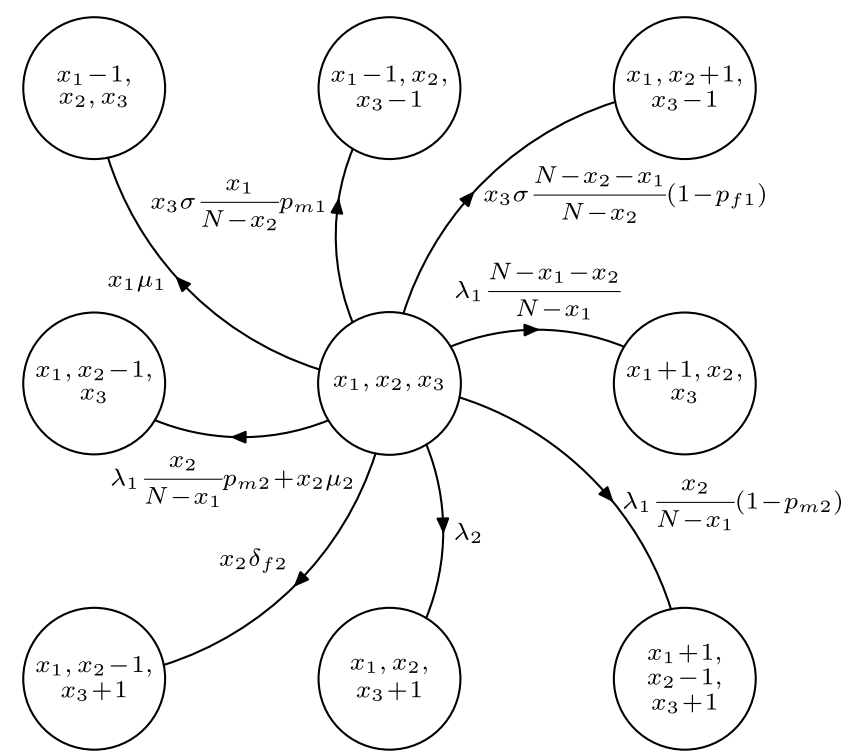

Fig. 2 Graph to illustrate the state transitions out of a system state $x=$ $\left(x_{1}, x_{2}, x_{3}\right)$ with $0<x_{1}<N, 0<x_{2}<N$ and $0<x_{3}<K$.

system state and to determine the corresponding transition rates, we first observe that a PU arrival occurs with rate $\lambda_{1}$. If in the current system state $x=\left(x_{1}, x_{2}, x_{3}\right)$ all channels are already occupied by other PUs (i.e., if $x_{1}=N$ ), the arriving PU is blocked and the system state remains unchanged. Therefore, with respect to changes in the system state due to the arrival of a PU, we only need to focus on the case where $x_{1}<N$. In such case, there are still $N-x_{1}$ channels available for the arriving PU and one of these available channels (either an idle channel or a channel in use by a SU) is randomly assigned to the PU. As such, a first event causing a change of the system state corresponds to the arrival of a PU at an idle channel. If $x_{1}<N$, the channel that is randomly assigned to the arriving PU will not be in use by a transmitting SU and, hence, be idle with probability $\frac{N-x_{1}-x_{2}}{N-x_{1}}$. In this case, the number of transmitting PUs in the system increases by one, whereas the numbers of transmitting and sensing SUs remain unchanged. Therefore, with rate $\lambda_{1} \frac{N-x_{1}-x_{2}}{N-x_{1}}$ we get a transition to state $y=\left(x_{1}+1, x_{2}, x_{3}\right)$ and hence,

$$
\begin{aligned}
q_{x, y} & =\lambda_{1} \frac{N-x_{1}-x_{2}}{N-x_{1}}, \\
& \text { if } y=\left(x_{1}+1, x_{2}, x_{3}\right), x_{1}<N .
\end{aligned}
$$

A second event then clearly corresponds to a PU arriving at a channel that is in use by a transmitting SU, which occurs with rate $\lambda_{1} \frac{x_{2}}{N-x_{1}}$ if $x_{1}<N$ and $x_{2}>0$ (i.e., there is at least one transmitting SU). In this case, the transmitting SU can correctly detect the PU arrival (with probability $1-p_{m 2}$ of having no class-B misdetection); the $\mathrm{SU}$ will then either evacuate the channel and go back to the sensing state if $x_{3}<K$ or leave the system if $x_{3}=K$. Important to note here is that the condition $x_{2}>0$ implies $x_{1}<N$, since $x_{1}+x_{2} \leq N$ (see (1)). The corresponding transition equations are then 


$$
\begin{aligned}
q_{x, y} & =\lambda_{1} \frac{x_{2}}{N-x_{1}}\left(1-p_{m 2}\right) \\
& \text { if } y=\left(x_{1}+1, x_{2}-1, x_{3}+1\right), x_{2}>0, x_{3}<K \\
q_{x, y} & =\lambda_{1} \frac{x_{2}}{N-x_{1}}\left(1-p_{m 2}\right) \\
& \text { if } y=\left(x_{1}+1, x_{2}-1, x_{3}\right), x_{2}>0, x_{3}=K
\end{aligned}
$$

Alternatively, still if $x_{2}>0$ (and $\left.x_{1}<N\right)$, the arriving PU is misdetected by the transmitting $\mathrm{SU}$ with probability $p_{m 2}$; the PU and SU then run into a collision and are both dropped out of the system; the state changes to $y=\left(x_{1}, x_{2}-1, x_{3}\right)$ in this case. Other events may, however, cause a similar state transition (see later), so we postpone giving the expression for the total transition rate to state $y=\left(x_{1}, x_{2}-1, x_{3}\right)$ for now.

A SU arrives with rate $\lambda_{2}$ and will enter the sensing state if the number of sensing SUs is less than $K$; otherwise, if $x_{3}=K$, the arriving SU cannot be accepted and gets blocked, and the system state remains unchanged. Therefore, with respect to changes in the system state due to the arrival of a SU, we only need to consider the case where $x_{3}<K$. We thus have

$$
q_{x, y}=\lambda_{2}, \quad \text { if } y=\left(x_{1}, x_{2}, x_{3}+1\right), x_{3}<K \text {. }
$$

A next event corresponds to the completion of a PU transmission, which can only occur if there is at least one transmitting PU (i.e., if $x_{1}>0$ ). Each of the $x_{1}$ transmitting PUs can complete transmission with rate $\mu_{1}$, and therefore

$$
q_{x, y}=x_{1} \mu_{1}, \quad \text { if } y=\left(x_{1}-1, x_{2}, x_{3}\right), x_{1}>0 \text {. }
$$

Another set of events is related to a transmitting SU leaving the transmitting state due to either transmission completion or a type-II false alarm. These events can only occur if there is at least one transmitting SU (i.e., if $x_{2}>0$ ). The completion of a SU transmission occurs with rate $x_{2} \mu_{2}$. With rate $x_{2} \delta_{f 2}$ a type-II false alarm occurs and in such case the transmitting SU stops transmission and either goes back to the sensing state if $x_{3}<K$ or leaves the system if $x_{3}=K$. As indicated above, a transmitting SU may also leave the transmitting state due to a PU arrival combined with a class-B misdetection, which occurs with rate $\lambda_{1} \frac{x_{2}}{N-x_{1}} p_{m 2}$ if $x_{2}>0$ (and $x_{1}<N$ ). These observations lead to the following transition equations:

$$
\begin{aligned}
q_{x, y} & =\lambda_{1} \frac{x_{2}}{N-x_{1}} p_{m 2}+x_{2} \mu_{2}, \\
& \text { if } y=\left(x_{1}, x_{2}-1, x_{3}\right), x_{2}>0, x_{3}<K ; \\
q_{x, y} & =\lambda_{1} \frac{x_{2}}{N-x_{1}} p_{m 2}+x_{2}\left(\mu_{2}+\delta_{f 2}\right), \\
& \text { if } y=\left(x_{1}, x_{2}-1, x_{3}\right), x_{2}>0, x_{3}=K ; \\
q_{x, y} & =x_{2} \delta_{f 2}, \\
& \text { if } y=\left(x_{1}, x_{2}-1, x_{3}+1\right), x_{2}>0, x_{3}<K .
\end{aligned}
$$

Finally, we look at system state changes corresponding to a sensing SU that finishes the sensing of a channel, which can only occur if there is at least one sensing SU (i.e., if $\left.x_{3}>0\right)$. Note that as SUs are aware of the channels occupied by other SUs (see Sect. 3), SUs will only sense the $N-x_{2}$ channels not already occupied by other SUs. Therefore, with respect to system state changes, we only need to consider the case where $x_{2}<N$. If $x_{3}>0$ and $x_{2}<N$, the event of a sensing SU finishing the sensing of a channel occurs with rate $x_{3} \sigma$. In case the (randomly picked) sensed channel is an idle channel and there was no type-I false alarm, the SU starts transmission and there is a transition to state $y=$ $\left(x_{1}, x_{2}+1, x_{3}-1\right)$. In case the sensed channel is occupied by a PU (which is only possible if $x_{1}>0$ ) and there was a class-A misdetection, the SU starts transmission, runs into a collision with the PU and both PU and SU are dropped out of the system; the state changes to $y=\left(x_{1}-1, x_{2}, x_{3}-1\right)$ in this case. Also note that $x_{1}>0$ implies $x_{2}<N$, in view of (1). All these observations then lead to

$$
\begin{aligned}
q_{x, y} & =x_{3} \sigma \frac{N-x_{2}-x_{1}}{N-x_{2}}\left(1-p_{f 1}\right), \\
& \text { if } y=\left(x_{1}, x_{2}+1, x_{3}-1\right), x_{2}<N, x_{3}>0 ; \\
q_{x, y} & =x_{3} \sigma \frac{x_{1}}{N-x_{2}} p_{m 1}, \\
& \text { if } y=\left(x_{1}-1, x_{2}, x_{3}-1\right), x_{1}>0, x_{3}>0 .
\end{aligned}
$$

For all other combinations of system states $x$ and $y$, with $x \neq y$, the transition rate $q_{x, y}$ from state $x$ to state $y$ equals 0 :

$$
q_{x, y}=0, \quad \text { otherwise. }
$$

Since the row sums of the transition rate matrix $Q$ of the CTMC equal zero, the diagonal elements $q_{x, x}$ of $Q$ are obtained from

$$
q_{x, x}=-\sum_{y \in S, y \neq x} q_{x, y} .
$$

The above equations completely determine the matrix $Q$. The row vector $\pi$ of steady-state probabilities of the CTMC can then be computed as the solution of the set of balance equations

$$
\pi Q=0,
$$

together with the normalization condition $\pi \mathbf{1}=1$, where $\mathbf{1}$ is a column vector of ones, or more explicitly

$$
\sum_{x_{1}=0}^{N} \sum_{x_{2}=0}^{N-x_{1}} \sum_{x_{3}=0}^{K} \pi_{\left(x_{1}, x_{2}, x_{3}\right)}=1 \text {, }
$$

where the element $\pi_{\left(x_{1}, x_{2}, x_{3}\right)}$ of vector $\pi$ denotes the steadystate probability that the system is in state $x=\left(x_{1}, x_{2}, x_{3}\right) \in$ $S$. Based on the vector $\pi$, we derive several performance measures in the next section.

\section{Performance Measures}

A first performance measure is the collision rate between 
PUs and SUs. The collision rate $\alpha=\alpha_{1}+\alpha_{2}$ is defined as the rate with which PUs and SUs are dropped out of the system because of collision, where $\alpha_{1}$ and $\alpha_{2}$ are related to a class-A and class-B misdetection respectively. These rates are calculated as follows:

$$
\begin{aligned}
& \alpha_{1}=\sum_{x_{1}=1}^{N} \sum_{x_{2}=0}^{N-x_{1}} \sum_{x_{3}=1}^{K} \pi_{\left(x_{1}, x_{2}, x_{3}\right)} x_{3} \sigma \frac{x_{1}}{N-x_{2}} p_{m 1}, \\
& \alpha_{2}=\sum_{x_{2}=1}^{N} \sum_{x_{1}=0}^{N-x_{2}} \sum_{x_{3}=0}^{K} \pi_{\left(x_{1}, x_{2}, x_{3}\right)} \lambda_{1} \frac{x_{2}}{N-x_{1}} p_{m 2} .
\end{aligned}
$$

In (16), the summation runs over all states $x=\left(x_{1}, x_{2}, x_{3}\right)$ in the state space $S$ of the CTMC with at least one transmitting $\mathrm{PU}$ and at least one sensing SU, in accordance with the fact that a class-A misdetection can only occur in such a system state (see transition equation (11) above). In a similar way, the summation in (17) runs over all states with at least one transmitting SU, as only for $x_{2}>0$ a class-B misdetection can occur (see transition Eqs. (7) and (8)).

The throughput of PUs $\eta_{\mathrm{PU}}$ is defined as the rate with which PUs successfully complete transmission, i.e., without collision. This rate can be computed as

$$
\eta_{\mathrm{PU}}=\sum_{x_{1}=1}^{N} \sum_{x_{2}=0}^{N-x_{1}} \sum_{x_{3}=0}^{K} \pi_{\left(x_{1}, x_{2}, x_{3}\right)} x_{1} \mu_{1},
$$

where the summation runs over all states in $S$ with $x_{1}>0$ in view of (6).

Similarly, the throughput of SUs $\eta_{\mathrm{SU}}$ is defined as the rate with which SUs successfully complete transmission, i.e., stop transmission not due to collisions or false alarms or interruptions upon a PU arrival. The throughput $\eta_{\mathrm{SU}}$ is hence given by

$$
\eta_{\mathrm{SU}}=\sum_{x_{2}=1}^{N} \sum_{x_{1}=0}^{N-x_{2}} \sum_{x_{3}=0}^{K} \pi_{\left(x_{1}, x_{2}, x_{3}\right)} x_{2} \mu_{2},
$$

where in the summation only states in $S$ with $x_{2}>0$ are considered in view of (7) and (8).

Finally, we are interested in obtaining the mean delay $E\left[d_{\mathrm{SU}}\right]$ experienced by a SU. To this end, we first calculate the mean number of transmitting SUs $E\left[n_{\mathrm{SU}, \mathrm{tr}}\right]$ and the mean number of sensing SUs $E\left[n_{\mathrm{SU}, \mathrm{se}}\right]$ as

$$
\begin{aligned}
& E\left[n_{\mathrm{SU}, \mathrm{tr}}\right]=\sum_{x_{2}=1}^{N} \sum_{x_{1}=0}^{N-x_{2}} \sum_{x_{3}=0}^{K} x_{2} \pi_{\left(x_{1}, x_{2}, x_{3}\right)}, \\
& E\left[n_{\mathrm{SU}, \mathrm{se}}\right]=\sum_{x_{1}=0}^{N} \sum_{x_{2}=0}^{N-x_{1}} \sum_{x_{3}=1}^{K} x_{3} \pi_{\left(x_{1}, x_{2}, x_{3}\right)} .
\end{aligned}
$$

The mean SU delay $E\left[d_{\mathrm{SU}}\right]$ then follows from Little's law as

$$
E\left[d_{\mathrm{SU}}\right]=\frac{E\left[n_{\mathrm{SU}, \mathrm{rr}}\right]+E\left[n_{\mathrm{SU}, \mathrm{se}}\right]}{\lambda_{2}(1-\gamma)},
$$

where $\gamma$ is the blocking probability of arriving SUs due to a full sensing room (i.e., $K$ sensing SUs in the system), which is given by

$$
\gamma=\sum_{x_{1}=0}^{N} \sum_{x_{2}=0}^{N-x_{1}} \pi_{\left(x_{1}, x_{2}, K\right)} .
$$

\section{Numerical Results and Discussion}

We investigate the performance of SUs and PUs in a CRN scenario with $N=20$ channels. The mean transmission times of SUs and PUs are $1 / \mu_{2}=1 / \mu_{1}=10 \mathrm{~ms}$. The offered PU load $\rho_{\mathrm{PU}}$ is defined as $\lambda_{1} /\left(\mu_{1} N\right)=0.3$ and the offered SU load is $\rho_{\mathrm{SU}}=\lambda_{2} /\left(\mu_{2} N\right)=0.5$. We consider a mean sensing time $1 / \sigma=10 \mathrm{~ms}$ and the maximum number of sensing SUs equals $K=50$. The aim of this section is a generic evaluation of the performance of a CRN and the impact of SUs on the primary network using a wide range of sensing error parameters. This way the big picture of the impact of imperfect spectrum sensing is revealed. Moreover, using our model a practitioner can conduct experiments to estimate the performance measures for different sets of parameters. The SU system can then be designed to ensure the specific QoS requirements of the PU network are met, i.e., to reach a SU interference level (collisions) with PUs that is allowed while maximizing the utilization of white spaces of the idle channels.

We start with exploring the effects of the class-A and class-B misdetection probabilities $p_{m 1}$ and $p_{m 2}$ on the collision rate $\alpha$ between SUs and PUs. Figure 3 shows curves of constant collision rate $\alpha$ versus $p_{m 1}$ and $p_{m 2}$. We set the values of $p_{f 1}$ and $\delta_{f 2}$ to zero. This models the case where the CRN does not experience false alarms. It can be seen from this figure that $\alpha$ is more sensitive to small values of $p_{m 1}$. In particular, it has been found that when $p_{m 1}$ increases in the interval $(0,0.2)$ the collision rate $\alpha$ rapidly increases. These observations are fully in line with Eq. (16) for $\alpha_{1}$. Clearly, in increase of $p_{m 1}$ will have a negative impact on the collision rate $\alpha_{1}$ for sensing SUs. As $p_{m 1}$ grows further, however, this negative impact of $p_{m 1}$ on $\alpha_{1}$ is expected to get somewhat

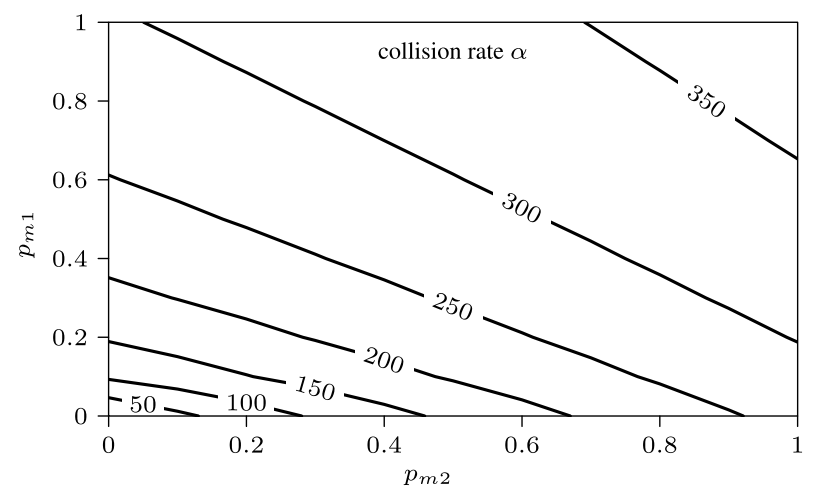

Fig. 3 Curves of constant collision rate $\alpha$ (collisions/s) versus the classA misdetection probability $p_{m 1}$ and the class-B misdetection probability $p_{m 2}$, for $1 / \sigma=1 / \mu_{2}=1 / \mu_{1}=10 \mathrm{~ms}, \rho_{\mathrm{PU}}=0.3, \rho_{\mathrm{SU}}=0.5, N=20$ and $p_{f 1}=\delta_{f 2}=0$. 


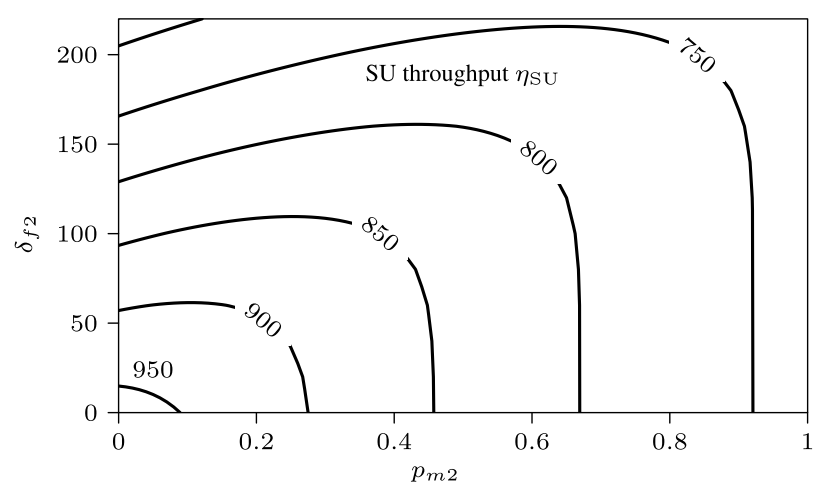

Fig. 4 Curves of constant throughput of SUs $\eta_{\mathrm{SU}}$ (SUs/s) versus the class-B misdetection probability $p_{m 2}$ and the type-II false alarm rate $\delta_{f 2}$, for $1 / \sigma=1 / \mu_{2}=1 / \mu_{1}=10 \mathrm{~ms}, \rho_{\mathrm{PU}}=0.3, \rho_{\mathrm{SU}}=0.5, N=20$ and $p_{f 1}=p_{m 1}=0$.

tempered by the decreasing number of sensing SUs due to collisions. Further numerical experiments, beyond those of Fig. 3, have also indicated that changing the mean sensing time $1 / \sigma$ between 1 and $20 \mathrm{~ms}$ only has negligible impact on $\alpha$. When the mean sensing time increases above $20 \mathrm{~ms}$, the value of $\alpha$ decreases, again in accordance with (16), while the qualitative behavior remains the same.

Figure 4 shows the effect of the class-B misdetection probability $p_{m 2}$ and the type-II false alarm rate $\delta_{f 2}$ of transmitting SUs on the throughput of SUs $\eta_{\mathrm{SU}}$, for $p_{f 1}=p_{m 1}=0$. We observe that for small values of $p_{m 2}<0.2$, an increase of $\delta_{f 2}$ strongly decreases $\eta_{\mathrm{SU}}$. For higher values of $p_{m 2}$, however, the influence of $\delta_{f 2}$ on the SU throughput decreases. For instance, for $p_{m 2}>0.9$, values of $\delta_{f 2}<140$ have no influence on $\eta_{\mathrm{SU}}$. A further increase of $\delta_{f 2}$ decreases the SU throughput for less than 5 percent. This observation can be explained by the fact that for higher values of $p_{m 2}$, an increasing number of SUs collide with PUs and are dropped out of the system. Likewise, for small values of $\delta_{f 2}<60$, an increase of $p_{m 2}$ decreases $\eta_{\mathrm{SU}}$. For higher values of $\delta_{f 2}>60, p_{m 2}$ has a smaller influence on $\eta_{\mathrm{SU}}$ for $p_{m 2}$ not too high. This is as expected since for higher values of $\delta_{f 2}$, more SUs stop transmitting due to a type-II false alarm and thus there are less chances for collisions with PUs. An interesting observation is that for $\delta_{f 2}>60$ and increasing $p_{m 2}, \eta_{\mathrm{SU}}$ somewhat increases until it reaches a maximum value and then $\eta_{\mathrm{SU}}$ decreases again forming a small peak. The increase in the first part can be explained by the fact that some PUs are dropped because of collisions, which makes it possible for some more SUs to utilize the channels.

The effect of $p_{m 2}$ and $\delta_{f 2}$ on the throughput of PUs $\eta_{\mathrm{PU}}$ is shown in Fig. 5. It can be seen that $p_{m 2}$ has a higher impact on the PU throughput than $\delta_{f 2}$, which is expected. Obviously, an increase of $p_{m 2}$ increases the collisions and consequently decreases $\eta_{\text {PU }}$. An increase of the type-II false alarm rate $\delta_{f 2}$ only slightly increases $\eta_{\mathrm{PU}}$, for all values of $p_{m 2}$.

Next, the effect of type-I false alarms and class-A misdetection errors by sensing SUs on the throughputs $\eta_{\mathrm{SU}}$ and

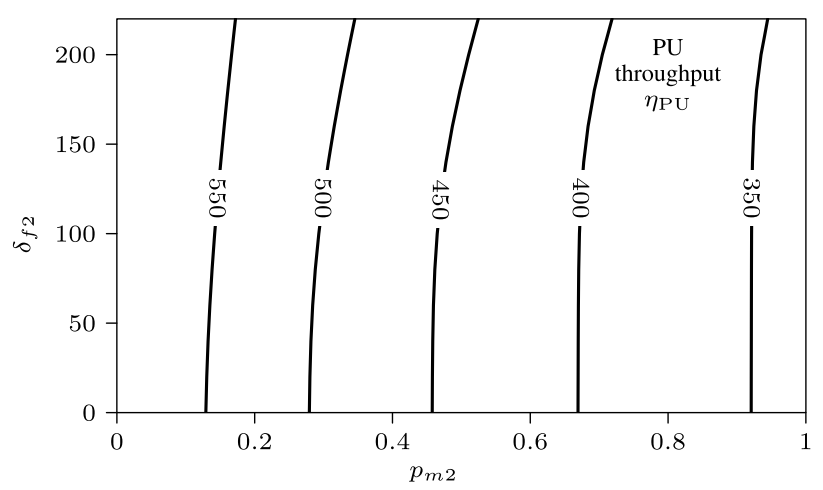

Fig. 5 Curves of constant throughput of PUs $\eta_{\mathrm{PU}}$ (PUs/s) versus the class-B misdetection probability $p_{m 2}$ and the type-II false alarm rate $\delta_{f 2}$, for $1 / \sigma=1 / \mu_{2}=1 / \mu_{1}=10 \mathrm{~ms}, \rho_{\mathrm{PU}}=0.3, \rho_{\mathrm{SU}}=0.5, N=20$ and $p_{f 1}=p_{m 1}=0$.

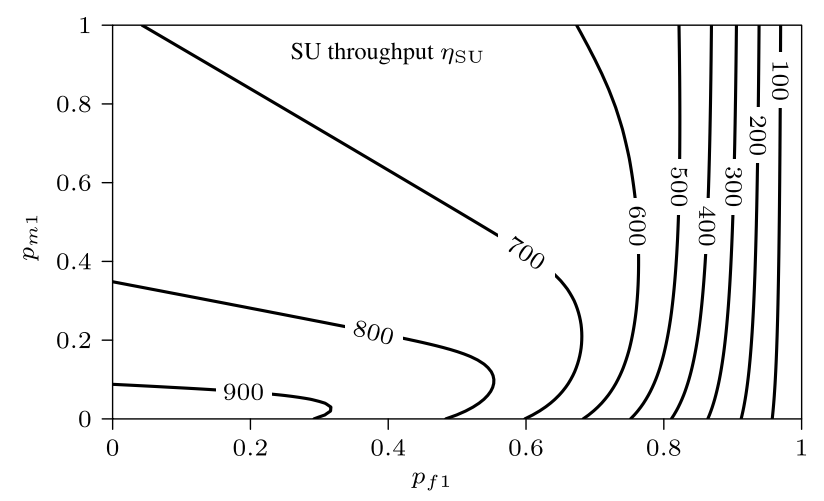

Fig. 6 Curves of constant throughput of SUs $\eta_{\mathrm{SU}}$ (SUs/s) versus the type-I false alarm probability $p_{f 1}$ and the class-A misdetection probability $p_{m 1}, 1 / \sigma=1 / \mu_{2}=1 / \mu_{1}=10 \mathrm{~ms}, \rho_{\mathrm{PU}}=0.3, \rho_{\mathrm{SU}}=0.5, N=20$ and $p_{m 2}=\delta_{f 2}=0$.

$\eta_{\mathrm{PU}}$ of secondary and primary users is illustrated in Figs. 6 and 7 respectively. Figure 6 gives curves of constant $\eta_{\mathrm{SU}}$ versus the probabilities $p_{f 1}$ and $p_{m 1}$, for $p_{m 2}=\delta_{f 2}=0$. It can be seen that for a given value of $p_{m 1}$, an increase of $p_{f 1}$ at first slowly decreases the throughput $\eta_{\mathrm{SU}}$ until $p_{f 1}$ is around 0.7. A further increase of $p_{f 1}$ then decreases $\eta_{\mathrm{SU}}$ sharply. The slower decrease for low $p_{f 1}$ is a consequence of the presence of enough sensing SUs to compete for the idle channels such that the event of some sensing SUs firing a false alarm has a more limited effect on the global SU throughput. As $p_{f 1}$ increases further, fewer sensing SUs are admitted to transmit and this starts to affect $\eta_{\mathrm{SU}}$ more strongly. Eventually, when $p_{f 1}=1, \eta_{\mathrm{SU}}=0$ and no sensing SUs are able to transmit. Another interesting observation is that for values of $p_{f 1}>0.5$, an increase of $p_{m 1}$ from 0 up to a certain point results in a higher throughput of SUs $\eta_{\mathrm{SU}}$. This can be explained by the fact that due to a higher probability of a class-A misdetection and the corresponding collision between a sensing SU and a PU, more and more PUs are dropped out of the system, which results in more white spaces being available for a sensing SU to start transmission.

Figure 7 shows the effect of the probabilities $p_{f 1}$ and 


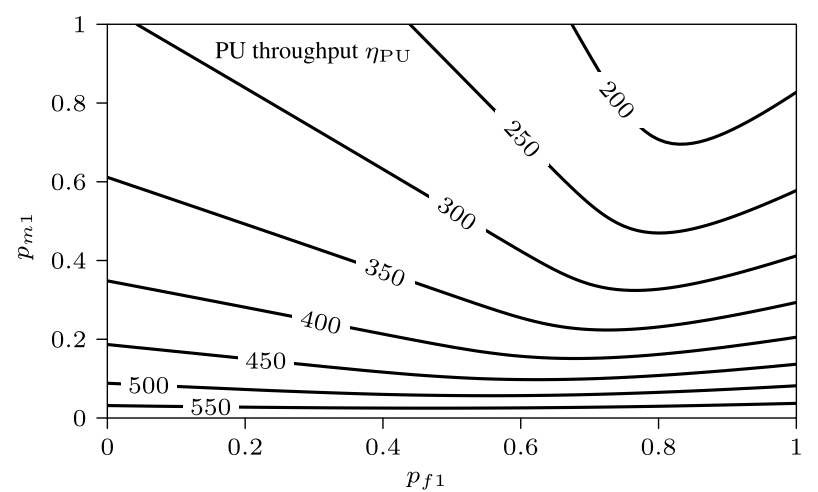

Fig. 7 Curves of constant throughput of PUs $\eta_{\mathrm{PU}}$ (PUs/s) versus the typeI false alarm probability $p_{f 1}$ and the class-A misdetection probability $p_{m 1}$, for $1 / \sigma=1 / \mu_{2}=1 / \mu_{1}=10 \mathrm{~ms}, \rho_{\mathrm{PU}}=0.3, \rho_{\mathrm{SU}}=0.5, N=20$ and $p_{m 2}=\delta_{f 2}=0$

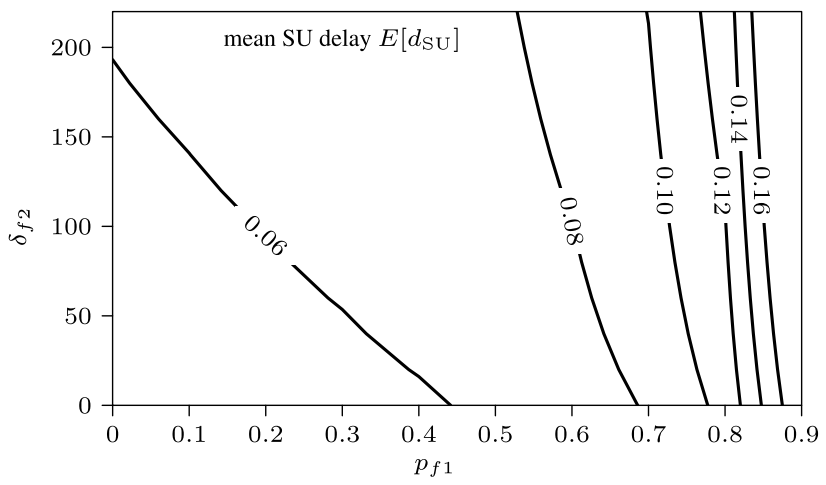

Fig. 8 Curves of constant mean SU delay $E\left[d_{\mathrm{SU}}\right]$ (s) versus the typeI false alarm probability $p_{f 1}$ and the type-II false alarm rate $\delta_{f 2}$, for $1 / \sigma=1 / \mu_{2}=1 / \mu_{1}=10 \mathrm{~ms}, \rho_{\mathrm{PU}}=0.3, \rho_{\mathrm{SU}}=0.5, N=20$ and $p_{m 1}=p_{m 2}=0$.

$p_{m 1}$ on the throughput of PUs $\eta_{\mathrm{PU}}$. From this figure, we observe the following. First, for low values of $p_{m 1}<0.1$, the probability of false alarm $p_{f 1}$ has a limited effect on $\eta_{\mathrm{PU}}$ and when $p_{m 1}=0$, this effect even disappears completely. This is as expected, since in a system without misdetection PUs do not experience any interference from SUs and therefore, the false alarm probability $p_{f 1}$ affects the performance of the SUs only. Second, for values of $p_{m 1}>0.1$, we notice that for increasing values of $p_{f 1}$, the throughput $\eta_{\mathrm{PU}}$ first decreases until a minumum value is reached and then increases again. The decrease of $\eta_{\mathrm{PU}}$ in the first part is due to the fact that for increasing $p_{f 1}$, more SUs stay in the sensing state, which increases the possibility of collisions with PUs. In the second part, the number of transmitting SUs sharply decreases (as mentioned above); hence, the probability that a sensing SU randomly selects a channel for sensing that is occupied by a

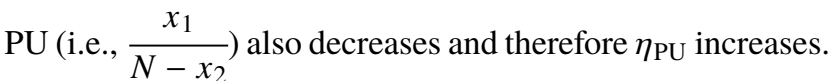

Figure 8 shows the mean SU delay as a function of the type-I false alarm probability $p_{f 1}$ and the type-II false alarm rate $\delta_{f 2}$, for $p_{m 1}=p_{m 2}=0$. We observe that the mean SU delay slowly increases as $p_{f 1}$ increases for low values of

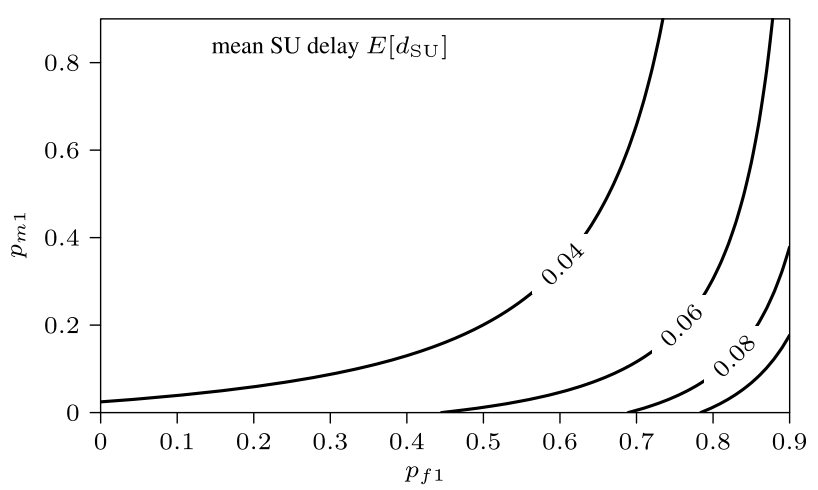

Fig. 9 Curves of constant mean SU delay $E\left[d_{\text {SU }}\right]$ (s) versus the type-I false alarm probability $p_{f 1}$ and the class-A misdetection probability $p_{m 1}$, $1 / \sigma=1 / \mu_{2}=1 / \mu_{1}=10 \mathrm{~ms}$, for $\rho_{\mathrm{PU}}=0.3, \rho_{\mathrm{SU}}=0.5, N=20$ and $p_{m 2}=\delta_{f 2}=0$.

$p_{f 1}$ and more rapidly increases for higher values of $p_{f 1}$. An increase of $\delta_{f 2}$ increases the mean SU delay further. It is also clear that $p_{f 1}$ has a higher influence on the mean SU delay than $\delta_{f 2}$.

Figure 9 illustrates the impact of the type-I false alarm probability $p_{f 1}$ and the class-A misdetection probability $p_{m 1}$ on the mean SU delay. It can be seen that an increase of $p_{m 1}$ between 0 and 0.9 leads to a decrease of the mean delay of around 50 percent. This is because for increasing $p_{m 1}$, the number of PUs in the system decreases due to collisions, thus leaving more white spaces for SUs. From our numerical experiments, it has also been found that sensing errors during a SU transmission (characterized by the parameters $p_{m 2}$ and $\delta_{f 2}$ ) have a similar qualitative influence on the mean SU delay as sensing errors by a sensing SU (as characterized by the parameters $p_{m 1}$ and $p_{f 1}$ ).

\section{Conclusion and Future Work}

In this paper, we developed a CTMC model of a CRN with sensing errors. The model takes into account false alarms and misdetection errors by both sensing and transmitting SUs. The impact of these errors on the throughput of PUs and SUs, the collision rate between PUs and SUs and the SU delay was thoroughly investigated. Future work will consider the extension to the case where the frequency channels do not have the same bandwidth. In this case, the complexity of the model and the state space of the corresponding CTMC will increase significantly.

\section{Acknowledgments}

This research has been partly funded by the Interuniversity Attraction Poles Programme initiated by the Belgian Science Policy Office.

\section{References}

[1] J. Mitola and G. Maguire, "Cognitive radio: Making software radios more personal," IEEE Pers. Commun., vol.6, no.4, pp.13-18, Aug. 
1999. DOI: $10.1109 / 98.788210$

[2] J. Marinho and E. Monteiro, "Cognitive radio: Survey on communication protocols, spectrum decision issues, and future research directions," Wireless Networks, vol.18, no.2, pp.147-164, Feb. 2012. DOI: $10.1007 / \mathrm{s} 11276-011-0392-1$

[3] B. Wang and K.J.R. Liu, "Advances in cognitive radio networks: a survey,” IEEE J. Sel. Topics Signal Process., vol.5, no.1, pp.5-23, Feb. 2011. DOI: 10.1109/JSTSP.2010.2093210

[4] L. Lu, X. Zhou, U. Onunkwo, and G.Y. Li, "Ten years of research in spectrum sensing and sharing in cognitive radio," EURASIP Journal on Wireless Communications and Networking, article no.28, pp.116, 2012. DOI: 10.1186/1687-1499-2012-28

[5] H. Sun, A. Nallanathan, C.-X. Wang, and Y. Chen, "Wideband spectrum sensing for cognitive radio networks: A survey," IEEE Wireless Commun., vol.20, no.2, pp.74-81, April 2013.

[6] N.T. Khajavi, S.S. Ivrigh, and S.M.S. Sadough, "A novel framework for spectrum sensing in cognitive radio networks," IEICE Trans. Commun., vol.E94-B, no.9, pp.2600-2609, Sept. 2011. DOI: 10.1587/transcom.E94.B.2600

[7] L. Lu, W. Jiang, H. Xiang, and W. Luo, "Adaptive spectrum sensing/transmission scheduling for cognitive radio," IEICE Trans. Commun., vol.E95-B, no.2, pp.635-638, Feb. 2012. DOI: 10.1587/transcom.E95.B.635

[8] E. Brockmeyer, H.L. Halstrom, and A. Jensen, The Life and Works of A.K. Erlang, The Copenhagen Telephone Company, Copenhagen, 1948

[9] S.M. Ross, Stochastic Processes, Second Edition, Wiley, New York, 1996.

[10] R.W. Wolff, Stochastic Modeling and the Theory of Queues, Prentice Hall, Englewood Cliffs, 1989.

[11] R. Durrett, Essentials of Stochastic Processes, Second ed., Springer, New York, 2012.

[12] J.R. Norris, Markov Chains, Cambridge University Press, Cambridge, 1997.

[13] L.-C. Wang, C.-W. Wang, and F. Adachi, "Load-balancing spectrum decision for cognitive radio networks," IEEE J. Sel. Areas Commun., vol.29, no.4, pp.757-769, April 2011. DOI: 10.1109/JSAC.2011.110408

[14] Y.-C. Liang, Y. Zeng, E.C.Y. Peh, and A.T. Hoang, "Sensingthroughput tradeoff for cognitive radio networks," IEEE Trans. Wireless Commun., vol.7, no.4, pp.1326-1337, April 2008. DOI: 10.1109/TWC.2008.060869

[15] S. Huang, X. Liu, and Z. Ding, "Optimal sensing-transmission structure for dynamic spectrum access," Proc. IEEE INFOCOM 2009, pp.2295-2303, Rio de Janeiro, Brazil, April 2009. DOI: 10.1109/INFCOM.2009.5062155

[16] Y. Gao and Y. Jiang, "Performance analysis of a cognitive radio network with imperfect spectrum sensing," Proc. IEEE INFOCOM 2010 Workshop on Cognitive Wireless Communications and Networking, pp.1-6, San Diego, USA, March 2010. DOI: 10.1109/INFCOMW.2010.5466711

[17] A.Ö. Ercan, "Analysis of asynchronous cognitive radio system with imperfect sensing and bursty primary user traffic," Signal, Image and Video Processing, vol.10, no.3, pp.593-600, March 2016. DOI: 10.1007/s11760-015-0782-4

[18] J.D. Kim and G. Hwang, "Cross-layer modeling and optimization of multi-channel cognitive radio networks under imperfect channel sensing," J. Industrial and Management Optimization, vol.11, no.3, pp.807-828, July 2015. DOI: $10.3934 /$ jimo.2015.11.807

[19] W. Cheng, X. Zhang, and H. Zhang, "Full-duplex spectrum-sensing and MAC-protocol for multichannel nontime-slotted cognitive radio networks,” IEEE J. Sel. Areas Commun., vol.33, no.5, pp.820-831, May 2015. DOI: 10.1109/JSAC.2014.2361078

[20] A. Homayounzadeh and M. Mahdavi, "Performance analysis of cooperative cognitive radio networks with imperfect sensing," Proc. International Conference on Communications, Signal Processing, and their Applications (ICCSPA 2015), pp.1-6, Sharjah, United Arab
Emirates, Feb. 2015. DOI: 10.1109/ICCSPA.2015.7081284

[21] X. Gelabert, O. Sallent, J. Perez-Romero, and R. Agusti, "Exploiting the operating point in sensing-based opportunistic spectrum access scenarios," Proc. IEEE International Conference on Communications (ICC 2009), pp.4152-4157, Dresden, Germany, Jun. 2009. DOI: $10.1109 /$ ICC.2009.5199018

[22] I. Suliman, J. Lehtomaki, T. Braysy, and K. Umebayashi, "Analysis of cognitive radio networks with imperfect sensing," Proc. 20th IEEE International Symposium on Personal, Indoor and Mobile Radio Communications, pp.1616-1620, Tokyo, Japan, Sept. 2009. DOI: 10.1109/PIMRC.2009.5450108

[23] I.M. Suliman, J.J. Lehtomaki, K. Umebayashi, and M. Katz, "Analysis of cognitive radio networks with imperfect sensing," IEICE Trans. Commun., vol.E96-B, no.6, pp.1605-1615, June 2013. DOI: 10.1587/transcom.E96.B.1605

[24] N. Tadayon and S. Aissa, "Modeling and analysis framework for multi-interface multi-channel cognitive radio networks," IEEE Trans. Wireless Commun., vol.14, no.2, pp.935-947, Feb. 2015. DOI: 10.1109/TWC.2014.2362535

[25] I.M. Suliman, J. Lehtomaki, and K. Umebayashi, "On the effect of false alarm rate on the performance of cognitive radio networks," EURASIP Journal on Wireless Communications and Networking, article no.244, 2015. DOI: 10.1186/s13638-015-0474-3

[26] S. Tang and Y. Xie, "Performance analysis of unreliable sensing for an opportunistic spectrum sharing system," International Journal of Communication Networks and Information Security, vol.3, no.3, pp.240-246, Dec. 2011.

[27] S. Tang, "A general model of opportunistic spectrum sharing with unreliable sensing," International Journal of Communication Systems, vol.27, no.1, pp.31-44, Jan. 2014. DOI: 10.1002/dac.2340

[28] O.I. Salameh, K. De Turck, H. Bruneel, C. Blondia, and S. Wittevrongel, "On the performance of secondary users in a cognitive radio network," Lecture Notes in Computer Science, vol.8499, pp.208-222, 2014. DOI: 10.1007/978-3-319-08219-6_15

[29] K.R. Chowdhury, M. Di Felice, and I.F. Akyildiz, "TP-CRAHN: A transport protocol for cognitive radio ad-hoc networks," Proc. IEEE INFOCOM 2009, pp.2482-2490, Rio de Janeiro, Brazil, April 2009. DOI: 10.1109/INFCOM.2009.5062176

[30] Y. Lu, D. Wang, and M. Fattouche, "Cooperative spectrum-sensing algorithm in cognitive radio by simultaneous sensing and BER measurements," EURASIP Journal on Wireless Communications and Networking, article no.136, May 2016. DOI: 10.1186/s13638-016$0635-\mathrm{z}$

[31] N. Tadayon and S. Aissa, "Multi-channel cognitive radio networks: modeling, analysis and synthesis," IEEE J. Sel. Areas Commun., vol.32, no.11, pp.2065-2074, Nov. 2014. DOI: 10.1109/JSAC.2014.1411RP06

[32] W. Afifi and M. Krunz, "Exploiting self-interference suppression for improved spectrum awareness/efficiency in cognitive radio systems," Proc. IEEE INFOCOM 2013, pp.1258-1266, Turin, Italy, April 2013. DOI: 10.1109/INFCOM.2013.6566918 


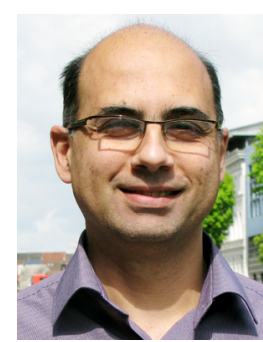

Osama Salameh was born in Schongau, Germany, in 1967. He received the M.Sc. degree and the Ph.D. degree in Computer Engineering from Odessa State Polytechnic University, Ukraine, in 1990 and 1996 respectively. During his career, he was employed by several universities in Palestine and Jordan. He is a member of the SMACS Research Group at Ghent University and a fulltime associate professor at the Arab American University-Jenin. His main research interests uation of telecommunication networks. include queueing models and performance eval-

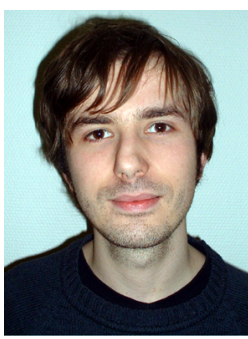

Koen De Turck obtained the M.Sc. degree in Engineering in 2004 and the Ph.D. degree in Computer Science Engineering in 2011, both from Ghent University, Belgium. Since 2015, he is an assistant professor at École CentraleSupélec, Université de Paris-Saclay, France. His current research interests include asymptotic and mean-field techniques, and their applications to telecommunication networks, large-scale games etc.

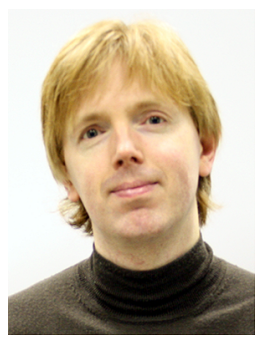

Dieter Fiems received the M.Sc. in Industrial Engineering from KaHo St-Lieven in 1997, a post-graduate degree in Computer Science from Ghent University in 1998 and the Ph.D. degree in Engineering from Ghent University in 2004. $\mathrm{He}$ is associate professor in the Department of Telecommunications and Information Processing at Ghent University. His current research interests include amongst others various applications of stochastic processes in the performance analysis of communication networks. Particularly, he is interested in applications of queueing theory and branching processes in wireless networks, optical networks and social networks.

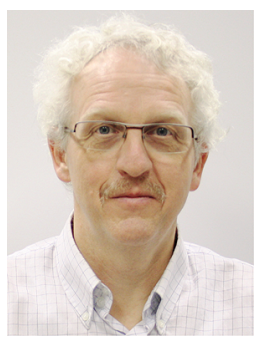

Herwig Bruneel was born in Zottegem, Belgium, in 1954. He received the master's degree in Electrical Engineering, the master's degree in Computer Science, and the Ph.D. degree in Computer Science in 1978, 1979 and 1984 respectively, all from Ghent University, Belgium. He is full professor in the Faculty of Engineering and Architecture and head of the Department of Telecommunications and Information Processing at the same university. He also leads the SMACS Research Group within this department. His main personal research interests include stochastic modeling and analysis of communication systems and discrete-time queueing theory. He is coauthor of the book H. Bruneel and B.G. Kim, "Discrete-Time Models for Communication Systems Including ATM" (Kluwer Academic Publishers, Boston, 1993). Since 2009, he holds a career-long Methusalem grant from the Flemish Government at Ghent University, specifically on Stochastic Modeling and Analysis of Communication Systems.

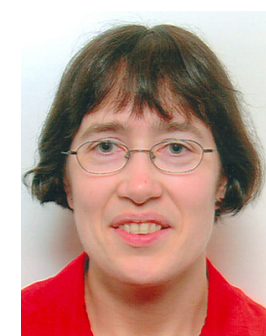

Sabine Wittevrongel was born in Gent, Belgium, in 1969. She received the master's degree in Electrical Engineering and the Ph.D. degree in Engineering from Ghent University, Belgium, in 1992 and 1998 respectively. In 1992, she joined the SMACS Research Group of the Department of Telecommunications and Information Processing at Ghent University. In 1998, she received the Scientific Award Alcatel Bell for the work entitled "Generic analytical techniques for the performance analysis of buffers in ATM networks". Since 2001, she is associate professor in the field of stochastic modeling and queueing analysis in the Faculty of Engineering and Architecture at Ghent University. Her main research interests include queueing theory, stochastic modeling and performance evaluation of communication networks, scheduling mechanisms and energy-efficient protocols. 